\title{
Correlation between Axial Length and Macular Thickness in Myopia
}

Mahmoud A. Rabea, Sayed M. Al sayed, Mohammad H. Abdel Zaher

Department of ophthalmology, Faculty of Medicine, Al-Azhar University, Cairo, Egypt

Corresponding author: Mohammad H. Abdel Zaher; Mobile: 01015048790; Email: drmohamadhanafy2@gmail.com

\begin{abstract}
Background: Myopia is the most common error of refraction and, in many countries; complications related to high myopia are a major cause of blindness. The prevalence of myopia has been reported as high as 70-90\% in some Asian countries, 30-40\% in Europe and the United States, and 10-20\% in Africa. Myopia, which is measured in diopters, has also been classified by degree. Low myopia usually describes myopia of -3.00 diopters or less. Medium myopia usually describes myopia between -3.00 and -6.00 diopters. High myopia usually describes myopia of -6.00 or more. Roughly $30 \%$ of myopes have high myopia.
\end{abstract}

Aim of the Work: To correlate macular thickness in the different degrees of axial myopia.

Subjects and Methods: Cross sectional study assessing 40 myopic eyes of 22 subjects. They were subdivided according to axial length into two groups: Group A (low myopes) 20 myopic eyes with their axial length between 24-26.5mm.Group B (high myopes) 20 myopic eyes with their axial length above $26.5 \mathrm{~mm}$. Cases were selected from the outpatient clinic of the Al-Azhar University Hospitals. All were above eighteen years of age. Each patient was subjected to a full clinical examination, refraction, axial length assessment by IOL master and macular thickness determined by Swept source OCT.

Results: The study revealed a highly significant negative correlation between axial length and macular thickness in all quadrants except foveal thickness which is non-significant positive correlation (an increase in thickness with increasing axial length). Macular thickness was significantly less in high myopes than low myopes except in the fovea. The spherical equivalent was significantly negatively correlated with increasing axial length.

Conclusion: In this study macular thickness in myopes was correlated with axial length. A significant negative correlation was found between increasing axial length and macular thickness in all quadrants of the macula except for the fovea. As the degree of myopia increased the average overall thickness of the macula decreased. No significant correlation was found between the foveal thickness and axial length.

Keywords: Myopia, axial length, macular thickness, OCT

\section{INTRODUCTION:}

Myopia is the most common error of refraction and, in many countries; complications related to high myopia are a major cause of blindness. The prevalence of myopia has been reported as high as $70-90 \%$ in some Asian countries, 30-40\% in Europe and the United States, and 10-20\% in Africa (1).

It is a refractive defect of the eye in which collimated light produces an image which comes into focus in front of the retina when accommodation is completely relaxed. Thus the image one sees comes to focus only on approximation of the object ${ }^{(2)}$.

Myopia, which is measured in diopters, has also been classified by degree. Low myopia usually describes myopia of -3.00 diopters or less. Medium myopia usually describes myopia between -3.00 and -6.00 diopters. High myopia usually describes myopia of -6.00 or more. Roughly $30 \%$ of myopes have high myopia ${ }^{(3)}$.

Similarly, myopia has also been classified according to the axial length (AL) into low to moderate myopia (AL $25.1 \pm$ $1.1 \mathrm{~mm})$ and high myopia (AL 27.1 $\pm 1.1 \mathrm{~mm})^{(4)}$

This refractive error can also be classified as pathological and nonpathological. Non-pathological myopia is also commonly referred to as simple myopia. In non-pathologic myopia the refractive structures of the eye develop within normal limits, however the refractive power of the eye does not correlate with the axial length. The degree of non-pathological myopia is usually minimal to moderate $(<6.00$ diopters $)$. 
Pathological myopia is generally classified as a high myopic refractive error that is progressive and generally presents very early in childhood. It is usually defined as spherical equivalent > 6.00 diopters or axial length $>26.5 \mathrm{~mm}^{(5)}$.

The macula is an oval-shaped highly pigmented yellow region near the center of the retina of the human eye. It has a diameter of around $5 \mathrm{~mm}$ and is often histologically defined as having two or more layers of ganglion_cells. Near its center is the fovea, a small pit that contains the largest concentration of cone cells in the eye and is responsible for central, high resolution vision. With the availability of modern imaging technologies, such as the Optical Coherence Tomography (OCT), in vivo measurement of retinal thickness has been made possible ${ }^{(6)}$.

\section{AIM OF THE WORK:}

To correlate macular thickness in the different degrees of axial myopia.

\section{SUBJECTS AND METHODS:}

This study was designed to be cross sectional assessing forty myopic eyes of twenty two subjects for correlation between the axial length and macular thickness. They were subdivided according to axial length into two groups:

- Group A (low myopes) which consists of 20 myopic eyes with their axial length between $24-26.5 \mathrm{~mm}$.

- Group B (high myopes) which consists of 20 myopic eyes with their axial length above $26.5 \mathrm{~mm}$.

Subjects were selected from the outpatient clinic of the Al-Azhar University Hospitals throughout the time period between January and April 2018. The study protocol was adhered to the tenets of the declaration of Helsinki and it was approved by the ethics board of Al-Azhar university. An informed written consent was taken from each participant in the study.

Each Subject was underwent a detailed ocular assesment. This assesment included:

- Un-corrected visual acuity, refraction (automated) and then correction using the spherical equivalent.
- Intraocular pressure will be determined by Goldmann Applanation Tonometry.

- Anterior segment examination by slit lamp biomicroscopy.

- Posterior segment examination by both indirect ophthalmoscopy and slit lamp with the ninety diopter auxillary lens.

\section{Inclusion criteria:}

-Age above eighteen years.

- Subjects with axial length above $24 \mathrm{~mm}$ measured by IOL master.

\section{Exclusion criteria:}

- Subjects with history of previous ocular surgery.

- Subjects with any ocular pathology such as glaucoma, cataract, diabetic retinopathy,......etc.

-Myopic changes in the fundus other than annular crescent, tigroid pattern or posterior staphyloma (not involving the macula).

- Macular pathology such as Fuch's spot, foveoschisis, or $\mathrm{CNV}, \ldots . .$. etc.

The axial lengths were determined by IOL master (Topcon ALADDIN HW2.0 SN: 74150190 which is found in Bab Al-Sharia university hospital).

Macular thickness was measured by Swept source OCT $1050 \mathrm{~nm}$ wave length (Topcon 3D OPTICAL COHERENCE TOMOGRAPHY DRI OCT Triton (plus) SN: 207100) which is found in Bab Al-Sharia university hospital. Macular thickness was measured in 9 regions. The central one millimeter was considered as the fovea. Extending from the central one millimeter to three millimeters was the parafoveal region and extending from three to five millimeters was the perifoveal region. Both the para and perifoveal regions were subdivided into four quadrants: superior, inferior, temporal and nasal.

\section{Statistical analysis:}

Recorded data were analyzed using the statistical package for social sciences, version 20.0 (SPSS Inc., Chicago, Illinois, USA). Quantitative data were expressed as mean \pm 
standard deviation (SD). Qualitative data were expressed as frequency and percentage.

\section{The following tests were done:}

- Independent-samples t-test of significance was used when comparing between two means.

- Paired sample t-test of significance was used when comparing between related samples.

- Chi-square $\left(\mathrm{x}^{2}\right)$ test of significance was used in order to compare proportions between two qualitative parameters.

- Pearson's correlation coefficient (r) test was used for correlating data.

- The confidence interval was set to $95 \%$ and the margin of error accepted was set to $5 \%$. So, the p-value was considered significant as the following:

- Probability (P-value)

- P-value >0.05 was considered insignificant.

- P-value $\leq 0.05$ was considered significant.

P-value $\leq 0.001$ was considered as highly significant.

\section{RESULTS:}

In this study a total of 22 cases (40 eyes) were assessed. These included 12 females and 10 males. They were divided according to axial length into group A (low myopes) and group B (high myopes). There were 20 eyes belonging to group $\mathrm{A}$ and 20 eyes to group B.
The age of group A ranged from 19 to 49 years (mean: $27.55 \pm 8.78$ ) and group $\mathrm{B}$ from 18 to 55 years $(33.33 \pm 11.02)$ (table 1$)$.

The refraction in group $A$ ranged from -3.25 to $-8.75(-5.86 \pm 1.49)$, and group B ranged from -9.25 to $-21.00(-14.11 \pm 3.40)$ (table2).

Axial lengths in group A ranged from 24.21 to $26.37 \mathrm{~mm}(25.15 \pm 0.65)$ and in group B from 26.53 to $31.73 \mathrm{~mm}(28.38 \pm 1.37)$ (table2).

There is no statistically significant difference between groups according to demographic data (Age, Gender) as shown in (table1).

There is highly statistically significant difference between groups according to spherical equivalent, axial lengths as shown in (table2).

Tables $(2,3,4)$ show the mean and standard deviation of macular thickness (fovea, parafovea, perifovea) in both groups. It also shows if the difference between the two groups is statistically significant ( $\mathrm{P}$ value). As shown:

There was statistically significant difference in parafoveal inferior quadrant, parafoveal nasal quadrant and perifoveal nasal quadrant thickness between the two groups. There is highly statistically significant difference in parafoveal superior quadrant, parafoveal temporal quadrant, perifoveal superior quadrant, perifoveal inferior quadrant and perifoveal temporal quadrant thickness between the two groups.

Table1: Comparison between groups according to demographic data.

\begin{tabular}{|l|c|c|c|c|}
\hline $\begin{array}{l}\text { Demographic } \\
\text { Data }\end{array}$ & $\begin{array}{c}\text { Group A } \\
(\mathbf{N}=11)\end{array}$ & $\begin{array}{c}\text { Group B } \\
(\mathbf{N}=12)\end{array}$ & t/x2\# & p-value \\
\hline $\begin{array}{l}\text { Age (years) } \\
\text { Mean } \pm \text { SD }\end{array}$ & $\begin{array}{c}27.55 \pm 8.78 \\
\text { Range }\end{array}$ & $\begin{array}{c}33.33 \pm 11.02 \\
18-55\end{array}$ & -1.384 & 0.181 \\
\hline $\begin{array}{l}\text { Gender } \\
\text { Female }\end{array}$ & $6(54.5 \%)$ & $7(58.3 \%)$ & & \\
Male & $5(45.5 \%)$ & $5(41.7 \%)$ & $0.136 \#$ & 0.934 \\
\hline
\end{tabular}


Table2: Comparison between groups according to spherical equivalent.

\begin{tabular}{|c|c|c|c|c|}
\hline & $\begin{array}{c}\text { Group A } \\
(\mathbf{N}=\mathbf{2 0})\end{array}$ & $\begin{array}{c}\text { Group B } \\
(\mathbf{N}=\mathbf{2 0})\end{array}$ & t-test & p-value \\
\hline $\begin{array}{l}\text { Spherical equivalent } \\
\text { Mean } \pm \text { SD } \\
\text { Range }\end{array}$ & $\begin{array}{l}-5.86 \pm 1.49 \\
-8.75 \_-3.25\end{array}$ & $\begin{array}{c}-14.11 \pm 3.40 \\
-21 \_-9.25\end{array}$ & 9.940 & $<0.001 * *$ \\
\hline $\begin{array}{l}\text { Axial length }(\mathbf{m m}) \\
\text { Mean } \pm \text { SD } \\
\text { Range }\end{array}$ & $\begin{array}{c}25.15 \pm 0.65 \\
24.21-26.37\end{array}$ & $\begin{array}{c}28.38 \pm 1.37 \\
26.53-31.73\end{array}$ & -9.536 & $<0.001 * *$ \\
\hline $\begin{array}{l}\text { Foveal thickness (um) } \\
\text { Mean } \pm \text { SD } \\
\text { Range }\end{array}$ & $\begin{array}{c}253.30 \pm 25.39 \\
189-295\end{array}$ & $\begin{array}{c}269.85 \pm 31.83 \\
211-321\end{array}$ & 1.818 & 0.077 \\
\hline
\end{tabular}

Table3: Comparison between groups according to parafoveal.

\begin{tabular}{|c|c|c|c|c|}
\hline Parafoveal & $\begin{array}{c}\text { Group A } \\
(\mathbf{N}=\mathbf{2 0})\end{array}$ & Group B $(\mathrm{N}=20)$ & t-test & p-value \\
\hline $\begin{array}{l}\text { Superior Quadrant Thickness } \\
\text { (um) } \\
\text { Mean } \pm \text { SD } \\
\text { Range }\end{array}$ & $\begin{array}{c}317.60 \pm 10.88 \\
290-334\end{array}$ & $\begin{array}{c}281.60 \pm 33.94 \\
200-326\end{array}$ & 4.518 & $<0.001 * *$ \\
\hline $\begin{array}{l}\text { Inferior Quadrant Thickness } \\
(\text { um) } \\
\text { Mean } \pm \text { SD } \\
\text { Range }\end{array}$ & $\begin{array}{c}312.05 \pm 15.69 \\
278-331\end{array}$ & $\begin{array}{c}287.00 \pm 36.54 \\
178-323 \\
\end{array}$ & 2.818 & $0.008 *$ \\
\hline $\begin{array}{l}\text { Nasal Quadrant Thickness (um) } \\
\text { Mean } \pm \text { SD } \\
\text { Range }\end{array}$ & $\begin{array}{c}315.10 \pm 19.88 \\
254-339\end{array}$ & $\begin{array}{c}292.15 \pm 42.44 \\
200-352\end{array}$ & 2.190 & $0.035^{*}$ \\
\hline $\begin{array}{l}\text { Temporal Quadrant Thickness } \\
\text { (um) } \\
\text { Mean } \pm \text { SD } \\
\text { Range }\end{array}$ & $\begin{array}{c}304.45 \pm 10.39 \\
281-322\end{array}$ & $\begin{array}{c}269.75 \pm 33.42 \\
190-308\end{array}$ & 4.434 & $<0.001 * *$ \\
\hline
\end{tabular}

Table4: Comparison between groups according to peri-foveal

\begin{tabular}{|l|c|c|c|c|}
\hline Peri-foveal & Group A (N=20) & Group B (N=20) & t-test & p-value \\
\hline $\begin{array}{l}\text { Superior Quadrant Thickness } \\
\text { (um) }\end{array}$ & & & & \\
$\begin{array}{l}\text { Mean } \pm S D \\
\text { Range }\end{array}$ & $276.50 \pm 12.15$ & $243.60 \pm 37.37$ & 3.744 & $<0.001^{* *}$ \\
\hline $\begin{array}{l}\text { Inferior Quadrant Thickness } \\
\text { (um) }\end{array}$ & $259-295$ & $146-291$ & & \\
$\begin{array}{l}\text { Mean } \pm S D \\
\text { Range }\end{array}$ & $279.35 \pm 20.78$ & $242.35 \pm 39.37$ & 3.717 & $<0.001^{* *}$ \\
\hline $\begin{array}{l}\text { Nasal Quadrant Thickness (um) } \\
\text { Mean } \pm S D\end{array}$ & $240-333$ & $172-303$ & & \\
Range & $290.20 \pm 33.28$ & $264.00 \pm 33.35$ & 2.487 & $0.017 *$ \\
\hline $\begin{array}{l}\text { Temporal Quadrant Thickness } \\
\text { (um) }\end{array}$ & $161-322$ & $207-316$ & \\
$\begin{array}{l}\text { Mean } \pm S D \\
\text { Range }\end{array}$ & $269.85 \pm 13.61$ & $227.85 \pm 31.47$ & 5.478 & $<0.001^{* *}$ \\
\hline
\end{tabular}


Table5: shows Correlation between axial length and other parameters in group A. There is significant negative correlation between axial length and parafoveal superior quadrant thickness. Also there is highly significant negative correlation between axial length and spherical equivalent.

Table5: Correlation between axial length and other parameters, using Pearson Correlation Coefficient in group A.

\begin{tabular}{|l|c|c|}
\hline \multirow{2}{*}{} & \multicolumn{2}{|c|}{ Axial length (mm) } \\
\cline { 2 - 3 } & r & p-value \\
\hline Spherical equivalent & -0.697 & \\
\hline Macular thickness & & $0.001^{* *}$ \\
\hline Foveal thickness (um) & 0.063 & 0.793 \\
\hline Parafoveal & & \\
\hline Superior Quadrant Thickness (um) & -0.441 & $0.049^{*}$ \\
\hline Inferior Quadrant Thickness (um) & -0.370 & 0.108 \\
\hline Nasal Quadrant Thickness (um) & -0.314 & 0.178 \\
\hline Temporal Quadrant Thickness (um) & -0.332 & 0.153 \\
\hline Peri-foveal & & \\
\hline Superior Quadrant Thickness (um) & -0.371 & 0.107 \\
\hline Inferior Quadrant Thickness (um) & -0.227 & 0.335 \\
\hline Nasal Quadrant Thickness (um) & -0.367 & 0.111 \\
\hline Temporal Quadrant Thickness (um) & -0.122 & 0.608 \\
\hline
\end{tabular}

In group B; Table 6 shows:

There is significant negative correlation between axial length and spherical equivalent, parafoveal superior quadrant thickness, parafoveal nasal quadrant thickness, parafoveal temporal quadrant thickness, perifoveal inferior quadrant thickness, perifoveal temporal quadrant thickness.
There is highly significant negative correlation between axial length and parafoveal inferior quadrant thickness, perifoveal nasal quadrant thickness.

There is non-significant negative correlation between axial length and foveal thickness, perifoveal superior quadrant thickness.

Table6: Correlation between axial length and other parameters, using Pearson Correlation Coefficient in group B.

\begin{tabular}{|l|c|c|}
\hline \multirow{2}{*}{} & \multicolumn{2}{c|}{ Axial length (mm) } \\
\cline { 2 - 3 } & r & $0.003^{*}$ \\
\hline Spherical equivalent & -0.636 & \\
\hline Macular thickness & & 0.346 \\
\hline Foveal thickness (um) & -0.222 & $0.004^{*}$ \\
\hline Parafoveal & & $<0.001^{* *}$ \\
\hline Superior Quadrant Thickness (um) & -0.610 & $0.004^{*}$ \\
\hline Inferior Quadrant Thickness (um) & -0.773 & $0.005^{*}$ \\
\hline Nasal Quadrant Thickness (um) & -0.617 & \\
\hline Temporal Quadrant Thickness (um) & -0.598 & 0.132 \\
\hline Peri-foveal & & $0.009^{*}$ \\
\hline Superior Quadrant Thickness (um) & -0.348 & $<0.001^{* *}$ \\
\hline Inferior Quadrant Thickness (um) & -0.570 & \multicolumn{2}{|c|}{} \\
\hline Nasal Quadrant Thickness (um) & -0.699 & \multicolumn{2}{|c|}{} \\
\hline
\end{tabular}


r- Pearson Correlation Coefficient

*p-value $<0.05 \mathrm{~S} ; * *$ p-value $<0.001 \mathrm{HS}$

Table7: shows the correlation between axial length and spherical equivalent, macular thickness in all patients. There is highly significant negative correlation between axial length and all parameters except foveal thickness which is non-significant positive correlation (an increase in thickness with increasing axial length).

Table7: Correlation between axial length and other parameters, using Pearson Correlation Coefficient in all patients.

\begin{tabular}{|l|c|c|}
\hline \multirow{2}{*}{} & \multicolumn{2}{|c|}{ Axial length (mm) } \\
\cline { 2 - 3 } & r & p-value \\
\hline Spherical equivalent & -0.899 & $<0.001^{* *}$ \\
\hline Macular thickness & & 0.093 \\
\hline Foveal thickness (um) & 0.209 & \\
\hline Parafoveal & & $<0.001^{* *}$ \\
\hline Superior Quadrant Thickness (um) & -0.751 & $<0.001^{* *}$ \\
\hline Inferior Quadrant Thickness (um) & -0.697 & $<0.001^{* *}$ \\
\hline Nasal Quadrant Thickness (um) & -0.569 & $<0.001^{* *}$ \\
\hline Temporal Quadrant Thickness (um) & -0.736 & \\
\hline Peri-foveal & & $<0.001^{* *}$ \\
\hline Superior Quadrant Thickness (um) & -0.598 & $<0.001^{* *}$ \\
\hline Inferior Quadrant Thickness (um) & -0.667 & $<0.001^{* *}$ \\
\hline Nasal Quadrant Thickness (um) & -0.595 & $<0.001^{* *}$ \\
\hline Temporal Quadrant Thickness (um) & -0.738 & \\
\hline
\end{tabular}

r- Pearson Correlation Coefficient

$*$ p-value $<0.05 \mathrm{~S}$; **p-value $<0.001 \mathrm{HS}$

\section{DISCUSSION:}

Myopia is the most common error of refraction and, in many countries; complications related to high myopia are a major cause of blindness. The prevalence of myopia has been reported as high as 70-90\% in some Asian countries, 30-40\% in Europe and the United States, and 10-20\% in Africa (1).

One of the most important of complications of myopia is the myopic macular hole ${ }^{(7)}$. For this reason our study has extensively studied myopic maculae and attempted to correlate them with several variables.
The relation between macular thickness and axial length in myopia, has been one of much interest over the past few years. Many researchers believe that the globe elongates in axial myopia, and the sclera stretches, so the macula must thin ${ }^{(4)}$.

Thus, multiple studies ${ }^{(6,5,8,9)}$ have been carried out in order to prove or disprove this hypothesis. All these studies have specific inclusion and exclusion criteria. They all exclude any ocular pathology and prior ocular surgeries. These studies have used various types of OCTs for assessment of the macular thickness and then further subdivided the macula into regions for comparison. Most studies used ultrasound biometry (A-scan) to determine the axial length. 
Our study included 40 eyes of 22 cases. Of these, there were 12 females and 10 males, ages ranging from 18 to 55years. They were divided according to axial length into low myopes (group A) and high myopes (group B). IOL master was used for axial length measurement. SS- OCT scan was used for macular thickness mapping.

Our study revealed that there was positive correlation between axial length and foveal thickness (an increase in thickness with increasing axial length) However, this correlation was statistically non-significant correlation (Table7). Also if we compared the thickness of the fovea in group A and group B, the group B had a greater thickness. However, this difference was non-significant.

In Ido et al. ${ }^{(6)}$ and Kim et al. ${ }^{(9)}$ the fovea was not correlated with increasing axial length.

In Chew et al. ${ }^{(5)}$ and Chan et al. ${ }^{(8)}$ there was also positive correlation between axial length and foveal thickness (an increase of foveal thickness with increase in axial length) but this correlation was found to be statistically significant.

In Chew et al. (5) the fovea was considered as the point of minimum thickness which would extend into what was presumed to be the parafoveal region. In our study it was considered as a region one millimeter in diameter. Therefore, our results cannot be compared to his results.

In Chan et al. ${ }^{(8)}$ the area considered to be the fovea was, as in our study, the central one millimeter. The reason that their results may have been significant while ours were not is that their study group included 143 eyes, which is much larger than our group (40 eyes).

The proposed theory for this finding (increasing foveal thickness with increasing axial length) may be due subclinical vitreoretinal traction not yet detected by OCT as in Chan et al. ${ }^{(8)}$. In Chew et al. ${ }^{(5)}$ the authors proposed that this increase in thickness may be due to pathologic subfoveal chorioretinal changes.

In our study the cause of nonsignificant correlation between the foveal thickness and axial length may be due to the small number of study patients. Also, the exclusion criteria eliminated any patient with any form of macular pathology. For instance, cases in which the posterior staphyloma or chorioretinal degenerations involved the macula were excluded. Patients with very high axial lengths often had macular lesions and therefore not included. Thus, our range of axial lengths was somewhat narrow.

In the remaining eight quadrants of the macula, our study found a highly significant negative correlation between axial length and the thickness (Table 9). In Chan et al. ${ }^{(8)}$ the significant negative correlation was found only in the perifoveal region and not in the parafoveal region. In Chew et al. ${ }^{(5)}$ there was statistically significant negative correlation in the parafoveal region, while in Kim et al. ${ }^{(9)}$ the significance was in the perifoveal region only. This finding was not similar to the study by Ido et al. ${ }^{(6)}$, in which there was no statistically significant change in retinal thickness with increasing axial length in any macular quadrants. In this study, contrary to the other ones, the OCT used was the Humphrey 2000, which is one of the first generation time domain OCTs. This may attribute to its different findings.

In our study when comparing the two groups (A and B), we found that thicknesses in all quadrants of the macula, except for the fovea, were less for high myopes than for low myopes. This finding was highly significant in all quadrants except in the in parafoveal nasal and inferior quadrants, perifoveal nasal quadrant in which the differences between the two groups was significant. In Ido et al. ${ }^{(6)}$ no significant difference was found among their three groups (emmetropes and low myopes, medium and high myopes). In Chan et al. ${ }^{(8)}$ there was significant difference between low and high myopes in the outer macular area only (thinner in high myopes). Other studies did not compare the thickness amongst subdivisions of myopes.

Our study revealed a highly significant negative correlation between axial length and subjective refraction (Table7). Thus, we can conclude that as the axial length increased the more highly negative spherical equivalent required for correction. This finding was also 
found in, Chew et al. ${ }^{(5)}$, Chan et al. ${ }^{(8)}$ and Kim et al. ${ }^{(9)}$.

As the spherical equivalent was highly significantly negatively correlated with axial length in our study, so there would be a similar significant correlation with all the macular quadrants as there was with the axial length.

Similar to our study, in Chan et al. ${ }^{(8)}$ total macular thickness decreased with increasingly negative spherical equivalent. No correlation was found between the degree of myopia and macular thickness in Chew et al.

\section{CONCLUSION:}

Myopia is the most common error of refraction and its pathological form may lead to severe visual impairment. It is subdivided into axial and refractive myopia. Axial myopia is due to elongation of the globe with backward displacement of the retina.

The OCT can accurately determine macular thickness overall and create a topography map of its regions.

In this study macular thickness in myopes was correlated with axial length. A significant negative correlation was found between increasing axial length and macular thickness in all quadrants of the macula except for the fovea. As the degree of myopia increased the average overall thickness of the macula decreased. No significant correlation was found between the foveal thickness and axial length.

The cases were subdivided into low and high myopes. The only significant correlations found were between the axial length and the macular thickness in two of the nine macular quadrants belonging to the group A. High myopes, in this study, had significantly thinner maculae than low myopes except for in the region of the fovea.

\section{REFERENCES:}

1. Cooper J, Schulman E, and Jamal $\mathbf{N}$ (2012): Current status on the development and treatment of myopia. Optometry, 83(5):179-99.

2. Russo, Francesco S, Mario $\mathrm{R}$ et al. (2014): myopia onset and progression; can it be prevented. International ophthalmology, 34(3):693-705.

3. Saw S, Gazzard G, Shih-Yen E et al. (2005): Myopia and associated pathological complications. Ophthalmic and Physological Optics, 25: 381-391.

4. Lam D, Leung K, Mohamed S et al. (2007): Regional variations in relationship between macular thickness measurements and myopia. Invest Ophthalmol Vis Sci., 48: 376-382.

5. Chew S, Foster P and Lim M (2005): Use of Optical Coherence Tomography to Assess Variations in Macular Retinal Thickness in Myopia. Invest Ophthalmol Vis Sci., 46: 974-978.

6. Ido $M$, Sugimoto $M$, and Sasoh $M$ (2003): Macular Thickness Measurements in Healthy Subjects with Different Axial Lengths Using Optical Coherence Tomography. The Journal of Retinal and Vitreous Diseases, 23:177-82.

7. Funata $M$, Morita $H$, and Tokoro $T$ (1995): A clinical study of the development of posterior vitreous detachment in high myopia. Retina, 15:117-24.

8. Chan B, Cheung $\mathbf{C}$, and Leung $\mathbf{K}$ (2007): Regional Variations in the Relationship between Macular Thickness Measurements and Myopia. Invest Ophthalmol Vis Sci., 48; 37682.

9. Kim C, Kim S, and Song W (2010): Macular Thickness Variations with Sex, Age, and Axial Length in Healthy Subjects: A Spectral Domain-Optical Coherence Tomography Study. Invest Ophthalmol Vis Sci., 51: 3913-9. 